\title{
A new synergistic relationship between xylan-active LPMO and xylobiohydrolase to tackle recalcitrant xylan
}

\author{
Anastasia Zerva ${ }^{1,2}$, Christina Pentari ${ }^{1}$, Sacha Grisel ${ }^{2}$, Jean-Guy Berrin ${ }^{2 *}$ and Evangelos Topakas ${ }^{1 *}$
}

\begin{abstract}
Background: Hemicellulose accounts for a significant part of plant biomass, and still poses a barrier to the efficient saccharification of lignocellulose. The recalcitrant part of hemicellulose is a serious impediment to the action of cellulases, despite the use of xylanases in the cellulolytic cocktail mixtures. However, the complexity and variety of hemicelluloses in different plant materials require the use of highly specific enzymes for a complete breakdown. Over the last few years, new fungal enzymes with novel activities on hemicelluloses have emerged. In the present study, we explored the synergistic relationships of the xylan-active AA14 lytic polysaccharide monooxygenase (LPMO), PCAA14B, with the recently discovered glucuronoxylan-specific xylanase TtXyn30A, of the (sub)family GH30_7, displaying xylobiohydrolase activity, and with commercial cellobiohydrolases, on pretreated natural lignocellulosic substrates.

Results: PCAA14B and TtXyn30A showed a strong synergistic interaction on the degradation of the recalcitrant part of xylan. PCAA14B was able to increase the release of xylobiose from TtXyn30A, showing a degree of synergism (DS) of 3.8 on birchwood cellulosic fibers, and up to 5.7 on pretreated beechwood substrates. The increase in activity was dose- and time- dependent. A screening study on beechwood materials pretreated with different methods showed that the effect of the PCAA14B-TtXyn30A synergism was more prominent on substrates with low hemicellulose content, indicating that PCAA14B is mainly active on the recalcitrant part of xylan, which is in close proximity to the underlying cellulose fibers. Simultaneous addition of both enzymes resulted in higher DS than sequential addition. Moreover, PCAA14B was found to enhance cellobiose release from cellobiohydrolases during hydrolysis of pretreated lignocellulosic substrates, as well as microcrystalline cellulose.
\end{abstract}

Conclusions: The results of the present study revealed a new synergistic relationship not only among two recently discovered xylan-active enzymes, the LPMO PCAA14B, and the GH30_7 glucuronoxylan-active xylobiohydrolase TtXyn30A, but also among PCAA14B and cellobiohydrolases. We hypothesize that PCAA14B creates free ends in the xylan polymer, which can be used as targets for the action of TtXYn30A. The results are of special importance for the design of next-generation enzymatic cocktails, able to efficiently remove hemicelluloses, allowing complete saccharification of cellulose in plant biomass.

Keywords: Synergism, AA14, GH30, Xylanase, Xylobiohydrolase, Lignocellulose

\footnotetext{
*Correspondence: jean-guy.berrin@inrae.fr; vtopakas@chemeng.ntua.gr ${ }^{1}$ Industrial Biotechnology and Biocatalysis Group, School of Chemical Engineering, National Technical University of Athens, 9 Iroon Polytechniou Str., Zografou Campus, 15780 Athens, Greece

${ }^{2}$ INRAE, Aix Marseille University, Biodiversité Et Biotechnologie Fongiques (BBF), UMR1 163, 13009 Marseille, France
}

\section{Background}

Recently, accumulating data on the discovery of novel enzyme activities tend to revolutionize the way microbial lignocellulose degradation was perceived during the last 30 years. Major breakthroughs in this field, such as the discovery of lytic polysaccharide monooxygenases 
(LPMOs, [1]) and more recently enzymes with xylobiohydrolase activity $[2,3]$ pave the way for major changes in the established theories regarding lignocellulose degradation. More specifically, for hemicellulose, the discovery of xylan-active AA14 LPMOs [4], as well as the conformations of xylan polymers interacting with cellulose [5-7], shed new light on the hemicelluloses structure and degradation pathways. According to a recent study, lytic xylan oxidases belonging to AA14 family selectively oxidize xylan polymers that cover cellulose fibrils, while no activity is detected on soluble xylan polymers, or underlying cellulose fibrils [4]. The explanation for this preference was based on recent discoveries regarding the conformations xylan polymers can receive when in contact with cellulose [6]. The authors hypothesized that AA14 LPMOs are active only on the twofold screw xylan polymers, acting as a shield of cellulose against microbial attack, in contrast with threefold screw soluble xylan polymers. The flat twofold screw conformation of xylan is recalcitrant to degradation by xylanase and displays a certain degree of 'crystallinity', which might be necessary for LPMO activity. This hypothesis is in line with earlier reports on the activity of cellulose-acting AA9 LPMOs on cellulose-associated xylan [8]. At the same time, Couturier et al. found that AA14 LPMOs produce only traces of C1-oxidized products, and therefore, the AA14 LPMO activity is detected only in synergism with a GH11 xylanase [4]. AA14 LPMOs also significantly boost the hydrolytic effect of cellulolytic cocktails of standard composition, on woody substrates.

Aside from AA14 LPMOs, which selectively oxidize recalcitrant xylan, AA9 LPMOs have been reported to target xylan associated to cellulose [8,9]. A single LPMO belonging to AA9 family has been reported to oxidize isolated xylans (PMO9A_MALCI, [10]), together with a bacterial AA10 LPMO (KpLPMO10A, [11]), although with different regioselectivity; KpLPMO10A released exclusively C4-oxidized sugars, while PMO9A_MALCI was shown to produce both $\mathrm{C} 1$ - and C4-oxidized products.

On the other hand, the recent discovery of the first fungal enzymes with xylobiohydrolase activity $[2,3]$ opens up new pathways for xylan degradation. GH30_7 xylanases not only act specifically on glucuronoxylan in an endo-manner, releasing uronic acid-decorated xylooligosaccharides (UXOS), but they can also act in an exomanner, releasing xylobiose [2, 3] from the non-reducing end, or xylose from the reducing end [12] of xylan polymers, or xylo-oligosaccharides (XOS). The recent discovery of such novel enzymatic activities raised numerous questions regarding the role and significance of such xylobiohydrolases in the decomposition of lignocellulose in natural ecosystems, and also regarding the potential of such enzymes in the design of biomass-degrading enzyme mixtures. Moreover, the activity of GH30_7 xylanases in natural woody substrates could shed light on the substrate preference and biological role of such novel enzymes.

Aside from the obvious significance of experimental data on single enzymes, the recalcitrant and complex structure of plant cell walls requires the coordinate action of a broad range of enzymes with varied specificities, acting synergistically, for the complete degradation of biomass. Therefore, the focus on enzyme combinations and their effects on lignocellulose is of equal importance. Enzyme synergism is the enhancement of substrate conversion observed through the combined action of two or more enzymes. Some enzyme combinations with strong synergistic effects are well established in the literature, such as the cellulases-xylanases synergism and the synergism among the cellulose-acting enzymes, such as CBHs and EGs $[13,14]$. Thus, it is widely accepted that xylan-degrading enzymes are indispensable in LCB degradation, because the removal of xylan increases the accessibility of cellulose-binding sites for cellulases [15, $16]$. On the other hand, the synergism between celluloseacting LPMOs and cellulases is well documented [13, 17, $18]$.

In the present work, the role of a xylan-active LPMO from Pycnoporus coccineus (PcAA14B) in the degradation of (hemi)cellulose was investigated through exploration of its synergistic relationships with a xylanase of the GH30_7 family (TtXyn30A) from Thermothelomyces thermophila (previously named Myceliophthora thermophila and Sporotrichum thermophile), and commercial cellobiohydrolases, on natural lignocellulosic substrates.

\section{Results}

\section{Synergism between PCAA14B LPMO and TtXyn30A xylanase}

As previously reported, PcAA14B LPMO acts synergistically with a family GH11 endoxylanase (AnXyn11) in the degradation of xylan-containing substrates [4]. In the present work, the synergism between $P c \mathrm{AA} 14 \mathrm{~B}$ and $T t$ Xyn30A was first studied on wood cellulosic fibers, containing $20 \%$ xylan, similarly to Couturier et al. [4]. The cellulosic fibers' substrate and the AnXyn11 xylanase were used as previously [4], to obtain comparable results. As shown in Fig. 1, the increase in XOS release was much higher in the case of TtXyn30A, compared to the synergistic action of AnXyn11 xylanase. More specifically, the increase in XOS release in the case of TtXyn30A was $338.6 \pm 13.7 \%$, corresponding to a degree of synergism (DS) $3.83 \pm 0.08$, while in the case of $A n X y n 11$, the increase was only $62.8 \pm 2.4 \%$, corresponding to a DS of $1.60 \pm 0.02$. However, the release of total oligosaccharides 

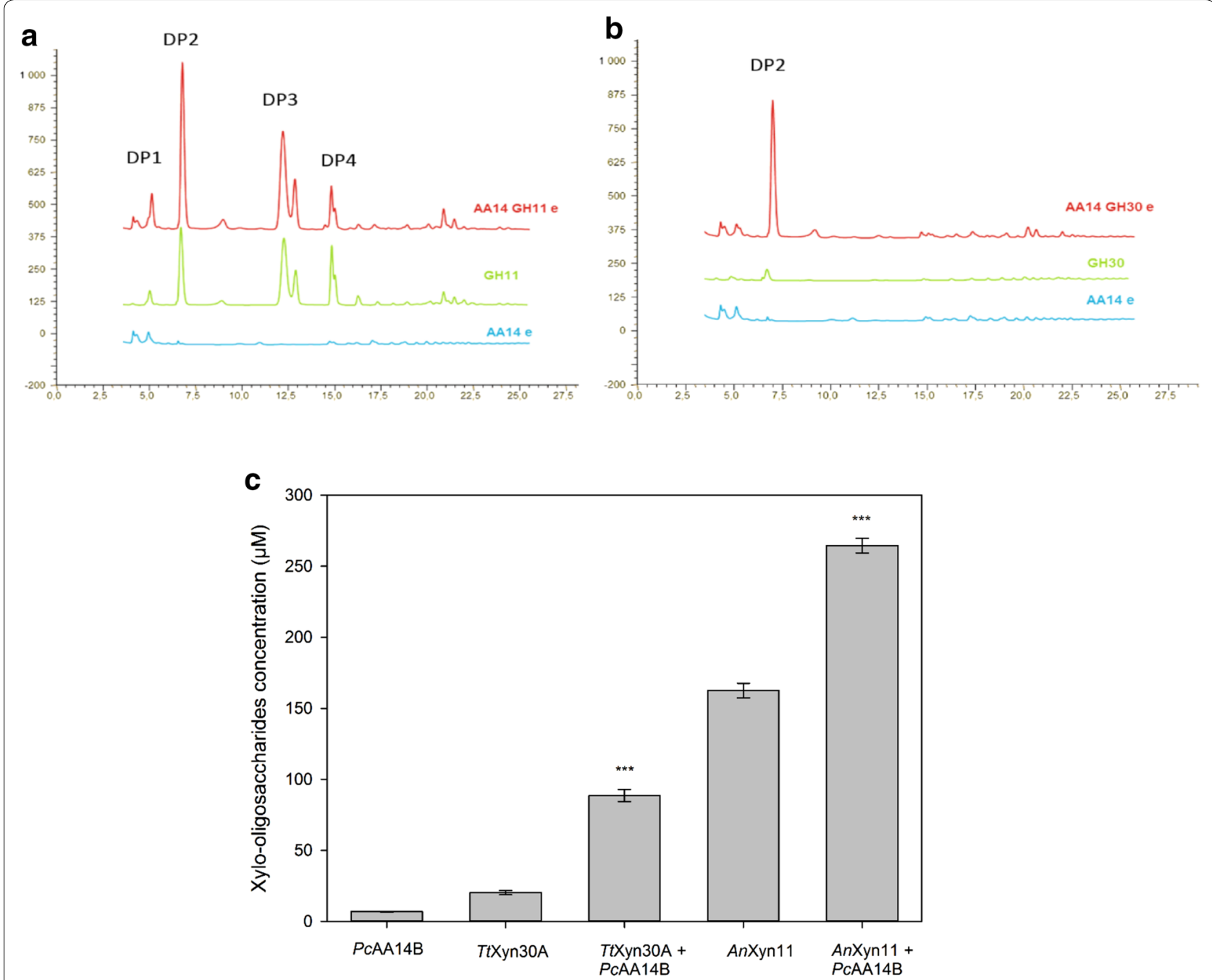

Fig. 1 HPAEC analysis of released XOS from cellulosic fibers, after incubation with a PCAA14A and AnXyn11 and b PCAA14A and TtXyn30A. Blue line: PCAA14A, green line: xylanase AnXyn11 or TtXyn30A, red line: PCAA14A together with xylanase AnXyn11 or TtXyn30A. c Xylo-oligosaccharides release from cellulosic fibers after 16-h incubation with each enzyme combination. Error bars represent the standard deviation from three independent experiments. Asterisks $\left({ }^{* *} \mathrm{p}<0.001\right)$ indicate significance based on Student's $t$ test

was much higher with $A n X y n 11$ (up to $264.5 \mu \mathrm{M}$, Additional file 1. Table S1) than with TtXyn30A (up to $88.6 \mu \mathrm{M}$, Additional file 1. Table S1), since the only xylooligosaccharide detected in the supernatant of TtXyn30A reactions was xylobiose.

To establish that the observed increase was indeed the result of a true synergistic relationship between PcAA14B and TtXyn30A, the next step was to investigate the release of soluble XOS over time. Of note, as the major product of TtXyn30A is xylobiose, all quantifications were based on xylobiose concentration. As shown in Fig. 2a, the release of xylobiose reaches a plateau already after $4 \mathrm{~h}$ of reaction with the use of
TtXyn30A alone, while with the addition of PcAA14B the release of xylobiose is constantly increasing up to $24 \mathrm{~h}$. This result is a strong indication that the action of PcAA14B is constantly creating new initiation sites for TtXyn30A. From the above results, it is strongly suggested that $T t$ Xyn30A alone is not capable of hydrolyzing the recalcitrant part of xylan.

In an attempt to gain further insight on the synergistic relationship between these two enzymes, we tested the effect of PcAA14B loading on the xylobiose release from TtXyn30A on cellulosic fibers. As shown in Fig. $2 \mathrm{~b}$, the addition of equimolar amount of PcAA14B to $T t X y n 30 \mathrm{~A}$ increased xylobiose release almost 

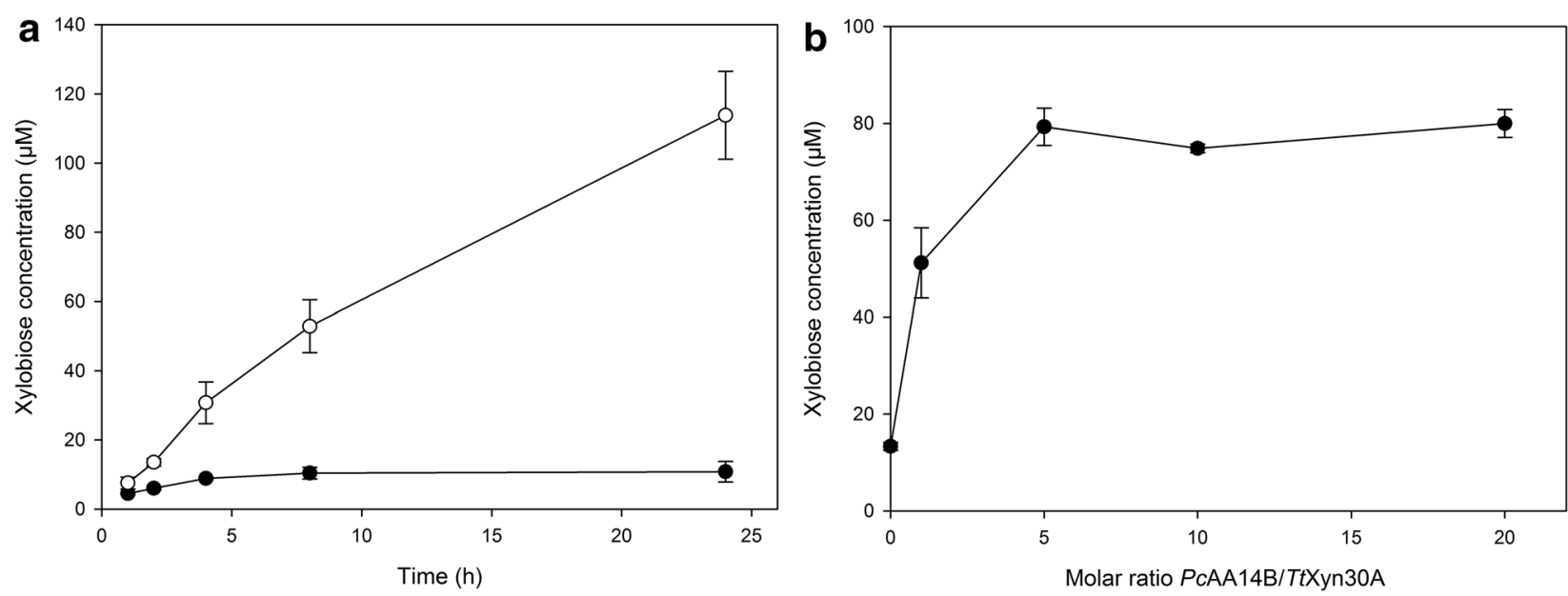

Fig. 2 a Time course of the xylobiose release by TtXyn30A (filled circles) and TtXyn30A with PCAA14B (open circles). b The effect of molar ratio of PCAA14B to TtXyn30A on xylobiose release. TtXyn30A was added at a final concentration of $0.1 \mu \mathrm{M}$. Error bars represent the standard deviation from three independent experiments

threefold, while increasing the molar ratio more than fivefold does not seem to increase further the synergy.

\section{Synergistic effects of PCAA14B LPMO and TtXyn30A xylanase on lignocellulosic substrates}

The synergism between PcAA14B LPMO and TtXyn30A xylanase was tested on different pretreated beechwood substrates. The substrates used in this study were selected based on their hemicellulose content, to gain better insights of the synergy of PcAA14B and TtXyn30A in the degradation of cellulose-associated xylan. Milox process (substrates 3, 5 and 7) leads to the delignification of the material, with the concomitant production of lignin degradation products [19]. On the other hand, acetone/water oxidation (substrates 1, 2, 4 and 6) is a recently developed methodology, offering the advantage of higher delignification, while producing fewer degradation products [19]. Moreover, the parameters, including acetone to water ratio, can be optimized for the removal of hemicelluloses and lignin. For example, higher acetone content led to substrates with higher hemicellulose content (substrates 4 and 6), in contrast with high water content, leading to substrates ( 1 and 2), where most of the hemicellulose is removed. Overall, the selected substrates were considered representative of a wide variety of compositions in terms of cellulose, hemicelluloses, and lignin content. The properties of the substrates and the pretreatment conditions are shown in Table 1, together with the release of xylobiose and the degree of synergism. Examples of the analysis of soluble XOS released from lignocellulosic substrates, after treatment with PcAA14B and TtXyn30A, are shown in Additional file 1: Fig. S1. During lignocellulosic substrates hydrolysis, xylobiose was the only product detected from the action of $T t X y n 30 \mathrm{~A}$, with HPAEC-PAD analysis. Therefore, all quantifications were made based on the exo-action of TtXyn30A, since uronic XOS were not detected in any case.

As shown in Table 1 and Additional file 1: Fig. S1, the increase in xylobiose release as a result of the synergism between PcAA14B and TtXyn30A varies a lot among the different substrates. The highest release of xylobiose by $T t$ Xyn30A was observed on a substrate with relatively high hemicellulose content (substrate 4, Table 1), but, at the same time, the enhancement of $T t X y n 30 \mathrm{~A}$ activity by PcAA14B was low in this case. On the contrary, the lowest xylobiose release was observed in the substrates 1,2 and 6 . The low hemicellulose content might explain the low concentration of released xylobiose from TtXyn30A on substrates 1 and 2. Despite the low activity of TtXyn30A on both substrates 1 and 2, the relative increase in xylobiose release was the highest observed (Table 1). Therefore, it seems that the hemicellulose remaining after pretreatment is the most recalcitrant to degradation, since the activity of TtXyn30A on this substrate is the lowest observed. On the other hand, the high increase in xylobiose release could be the result of a high LPMO activity, and therefore, this recalcitrant part of hemicellulose seems to be an ideal substrate for PcAA14B.

The synergistic activity of $P c A A 14 \mathrm{~B}$ and TtXyn30A seems to be interrupted in the case of substrates 6 and 7. Those are the substrates with the highest hemicellulose content tested in this study. The apparent loss of PcAA14B activity on these substrates is a further indication of the substrate preference of the enzyme; high content of residual hemicellulose indicates that possibly the 
Table 1 Results on the synergism between PCAA14B and TtXyn30A on different lignocellulosic substrates

\begin{tabular}{|c|c|c|c|c|c|c|c|c|}
\hline Substrate \# & $\begin{array}{l}\text { Pretreatment } \\
\text { conditions }\end{array}$ & $\begin{array}{l}\text { Cellulose } \\
(\% \mathrm{w} / \mathrm{w})\end{array}$ & $\begin{array}{l}\text { Acid-insoluble } \\
\text { lignin }(\% \mathrm{w} / \mathrm{w})\end{array}$ & $\begin{array}{l}\text { Acid-soluble } \\
\text { lignin }(\% \mathrm{w} / \mathrm{w})\end{array}$ & $\begin{array}{l}\text { Hemicellulose } \\
(\% \mathrm{w} / \mathrm{w})\end{array}$ & $\begin{array}{l}\text { TtXyn30A } \\
(\mu \mathrm{M} \\
\text { Xylobiose) }\end{array}$ & $\begin{array}{l}\text { PcAA14B + } \\
\text { TtXyn30A ( } \mu \mathrm{M} \\
\text { xylobiose) }\end{array}$ & $\begin{array}{l}\text { Degree } \\
\text { of synergism }\end{array}$ \\
\hline 1 & $\begin{array}{l}\text { d. } \mathrm{H}_{2} \mathrm{O} / \text { acetone } \\
(50 / 50 \%), \text { Air } \\
\left(40 \% \mathrm{O}_{2}\right), 8.5 \\
\text { bar }\left(175^{\circ} \mathrm{C}, 2 \mathrm{~h}\right)\end{array}$ & 79.47 & 8.4 & 0.5 & 4.01 & $1.23 \pm 0.00$ & $7.00 \pm 0.30(* * *)$ & $5.70 \pm 0.20$ \\
\hline 2 & $\begin{array}{l}\text { d. } \mathrm{H}_{2} \mathrm{O} / \text { acetone } \\
(75 / 25 \%), \text { Air } \\
\left(40 \% \mathrm{O}_{2}\right), 40 \\
\text { bar, } 175^{\circ} \mathrm{C}\end{array}$ & 84.10 & 11.5 & 1.0 & 5.18 & $0.98 \pm 0.06$ & $2.30 \pm 0.07\left(^{* * *}\right)$ & $2.35 \pm 0.07$ \\
\hline 3 & $\begin{array}{l}\text { Milox } 80^{\circ} \mathrm{C}, 1 \mathrm{~h}, \\
\text { formic acid }\end{array}$ & 80.30 & 6.3 & 1.0 & 12.35 & $26.7 \pm 1.80$ & $32.3 \pm 1.3\left(^{*}\right)$ & $1.21 \pm 0.03$ \\
\hline 4 & $\begin{array}{l}\text { d. } \mathrm{H}_{2} \mathrm{O} / \text { acetone } \\
(25 / 75 \%), \text { Air } \\
\left(40 \% \mathrm{O}_{2}\right), 40 \\
\text { bar, } 175^{\circ} \mathrm{C}\end{array}$ & 75.36 & 2.1 & 1.6 & 17.93 & $36.1 \pm 1.20$ & $46.1 \pm 2.3\left({ }^{* *}\right)$ & $1.28 \pm 0.10$ \\
\hline 5 & $\begin{array}{l}\text { Milox } 80^{\circ} \mathrm{C}, 1 \mathrm{~h} \text {, } \\
\text { formic acid, } \\
1 \mathrm{bar}\end{array}$ & 75.31 & 2.1 & 1.2 & 19.21 & $13.9 \pm 1.10$ & $17.2 \pm 1.1\left(^{*}\right)$ & $1.24 \pm 0.04$ \\
\hline 6 & $\begin{array}{l}\text { d. } \mathrm{H}_{2} \mathrm{O} / \text { acetone } \\
\text { (25/75\%), Air, } \\
20 \text { bar }\end{array}$ & 50.22 & 15.7 & 3.0 & 19.71 & $3.10 \pm 0.25$ & $2.20 \pm 0.00\left(^{*}\right)$ & - \\
\hline 7 & $\begin{array}{l}\text { Milox } 80^{\circ} \mathrm{C}, 3 \mathrm{~h}, \\
\text { acetic acid }\end{array}$ & 51.84 & 4.6 & 3.0 & 29.06 & $20.1 \pm 1.00$ & $18.0 \pm 0.8$ (ns) & - \\
\hline
\end{tabular}

The data on the pretreatment conditions and compositional analyses have been transferred by [19]. Error bars represent the standard deviation from three independent experiments. Asterisks $\left({ }^{*} \mathrm{p}<0.1 ;{ }^{* *} \mathrm{p}<0.01 ;{ }^{* * *} \mathrm{p}<0.001\right.$ ) indicate significance based on Student's $t$ test.

ns not significant

recalcitrant part of xylan, which is the assumed preferred substrate for PcAA14B, is not accessible by the enzyme. Substrate 6 contains much more residual lignin, which may inhibit the performance of TtXyn30A due to its adsorption. To test this hypothesis, we performed a laccase treatment in substrate 6 , aiming at the removal of residual lignin. The results showed that laccase treatment of substrate 6 increased xylobiose release from $T t$ Xyn30A by $19.7 \pm 2.9 \%$, indicating that residual lignin is responsible, at least to a certain extent, for TtXyn30A inhibition.

Products of the endoxylanase activity of TtXyn30A, such as 4-O-MeGlcA decorated XOS, were not detected in the above conditions. This could not only be attributed to their low concentration in the experimental setup of the present study, or to their low abundance in the beechwood substrates, but it could also indicate that the endo-action of $T t \mathrm{Xyn} 30 \mathrm{~A}$ is inhibited by the structure of the recalcitrant twofold xylan that is bound with the underlying cellulose fibrils. In this case, the role of PcAA14B could be much more crucial. To elucidate this, hydrolysis of substrates 1 and 4 was performed with increased concentration of TtXyn30A, in the absence of PcAA14B. Positive controls with soluble beechwood xylan were also included. The samples were properly diluted and analyzed with HPAEC-PAD, and the chromatograms are shown in Additional file 1: Fig. S2.
In the samples of substrate 1 , no UXOS were detected, which can be explained by the low hemicellulose content of this substrate. On the other hand, in soluble beechwood substrate, UXOS were detected, and one of them was identified as 2,3-(4-O-methyl- $\alpha$-D-glucuronyl)xylotriose, against a suitable standard. In samples of substrate 4 , one small peak appeared, with retention time corresponding to a possible UXOS product. The same peak was also detected in soluble beechwood xylan samples (Additional file 1: Fig. S2). This result corroborates with the higher hemicellulose content of substrate 4 and the higher activity of TtXyn30A, compared with the other tested substrates.

\section{Effect of sequential or simultaneous addition of enzymes on the hydrolytic reactions}

Synergistic relationships among lignocellulose-acting enzymes are often time-dependent, and the order of enzyme addition can offer valuable insights regarding their mode of action on their polymer substrate. The effect of sequential or simultaneous addition of PcAA14B and $T t \mathrm{Xyn} 30 \mathrm{~A}$ was examined, and the results are shown in Fig. 3. The maximum release of xylobiose was shown when both enzymes were added simultaneously. In this case, the DS was found $1.96 \pm 0.30$ for the first $24 \mathrm{~h}$, and $2.70 \pm 0.09$ when the reaction was prolonged for 


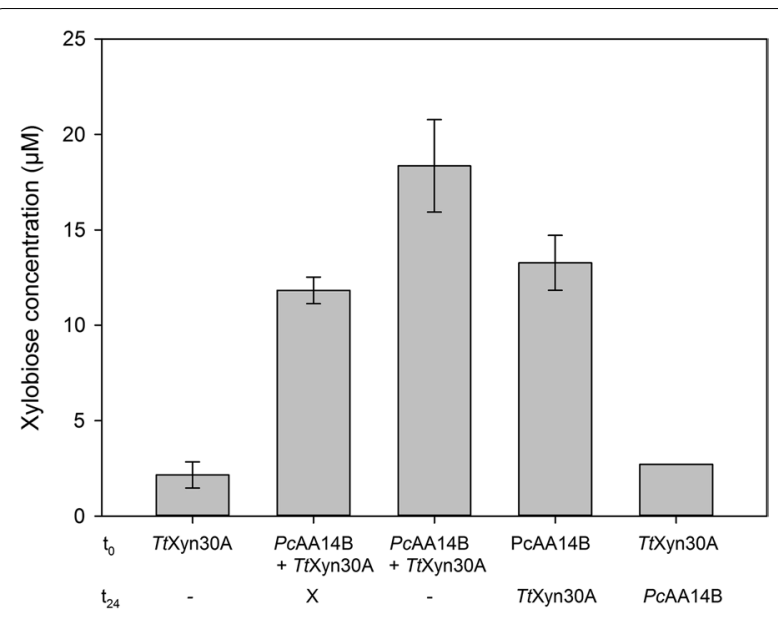

Fig. 3 Time dependence of the synergism between PCAA14A and TtXyn30A. The enzymes and their combinations were added at the specified time points, after boiling the reaction mixture to deactivate the first enzyme. The reaction was performed with substrate 1 , and it was stopped at $48 \mathrm{~h}$, except $\mathrm{X}$, where the reaction was stopped at $24 \mathrm{~h}$. Error bars represent the standard deviation from three independent experiments

$48 \mathrm{~h}$. When PcAA14B was added first for $24 \mathrm{~h}$ prior to the addition of TtXyn30A, the DS was $1.93 \pm 0.02$, similarly to the case where both enzymes were added together and kept for $24 \mathrm{~h}$. When TtXyn30A was added first for $24 \mathrm{~h}$ prior to the addition of PcAA14B, no synergism was observed, and the xylobiose release reached $2.70 \pm 0.00 \mu \mathrm{M}$ (Fig. 3). This result is expected, since PcAA14B does not release soluble products in detectable quantities. Therefore, an additional experiment was performed, where the substrate was pretreated with TtXyn30A and after $24 \mathrm{~h}$, PcAA14B was added, without deactivating the xylanase. The results were surprising, since the concentration of xylobiose release $(2.80 \pm 0.24 \mu \mathrm{M})$ was similar to the reaction when the xylanase was deactivated before LPMO addition. To determine the effect of enzyme stability to the above results, stability tests were performed for both enzymes under the same conditions. Both enzymes were found to be stable at $4{ }^{\circ} \mathrm{C}$ for $48 \mathrm{~h}$, while TtXyn30A was also found stable at $45{ }^{\circ} \mathrm{C}$ during $48 \mathrm{~h}$ in the absence of substrate. PcAA14b was found to maintain its activity fully for the first $24 \mathrm{~h}$, but up to $48 \mathrm{~h}$ the residual activity drops to $55.9 \pm 1.4 \%$. The stability of $T t$ Xyn30A was also tested in the presence of substrate 1 . Surprisingly, after $24 \mathrm{~h}$ at $45^{\circ} \mathrm{C}$, the residual activity of TtXyn30A dropped to $9.00 \pm 0.57 \%$ of the initial, while after $48 \mathrm{~h}$ only traces of the initial activity were detected in the supernatant. This result could be due to unproductive binding of TtXyn30A to the substrate and specifically to the lignin part, which in substrate 1 amounts to $8.9 \%(\mathrm{w} / \mathrm{w})$. This finding is in accordance with our results regarding the diminished activity of $T t \mathrm{Xyn} 30 \mathrm{~A}$ in substrate 6 due to its high lignin content. Therefore, when $T t$ Xyn30A is added first, it is possible that the enzyme is already "deactivated" due to unproductive binding on lignin prior to PcAA14B addition.

\section{Synergistic effects of PcAA14B and cellobiohydrolases on the hydrolysis of lignocellulosic substrates}

The next step was to explore the synergistic relationships of PcAA14B with single cellulose-acting enzymes, since in our previous study, the increase of released glucose by a complex (hemi)cellulolytic cocktail by the addition of PcAA14B was documented [4]. The synergism between PcAA14B and cellobiohydrolases CBHI and CBHII was tested on substrate 1 (Table 1), since maximum PcAA14B activity was observed using this substrate, in the previous experiments with TtXyn30A. The quantification of the results was based on cellobiose release, and the results are shown in Fig. 4a. PcAA14B, although it is not active on cellulose, was shown to greatly increase the release of cellobiose by $\mathrm{CBH}$ from substrate 1 , even though peaks corresponding to oxidized sugars, or xylo-oligomers, were not detected. Although the release of cellobiose was more prominent in the reaction of PcAA14B with CBHI, the DS was slightly higher in combination with CBHII $(1.58 \pm 0.15$ and $1.87 \pm 0.01$, respectively). The synergism between PcAA14B and CBHII was further tested on microcrystalline cellulose (Avicel), with or without cysteine as electron donor, and the results are shown in Fig. 4b. The addition of PcAA14B enhanced the production of cellobiose from CBHII, with or without the addition of electron donor, corresponding to DS of $3.70 \pm 0.35$ and $3.35 \pm 0.40$, respectively.

\section{Discussion}

Regarding the enzymatic degradation of lignocellulosic materials, the 'blocking effect' of xylan on the accessibility of the underlying cellulose to cellulases is widely established in literature [20]. This is further supported by the number of studies on the synergistic effects of xylanases on cellulose hydrolysis by cellulases [15, 16, 21, 22] . However, a large part of xylan is still recalcitrant to degradation by most known enzymes, leading research not only toward the discovery of new enzymatic activities, but also on the exploration of new synergistic interactions among known enzymes [20]. In the present work, not only the synergistic interactions between the xylanactive LPMO PcAA14B and the glucuronoxylan-specific xylobiohydrolase $T t$ Xyn30A, but also cellobiohydrolases, fill a significant knowledge gap on our understanding of the microbial degradation of (hemi)cellulose.

Both AA14 and GH30_7 are enzyme classes found in fungal saprotrophs [2, 4]. Bioinformatics analyses 

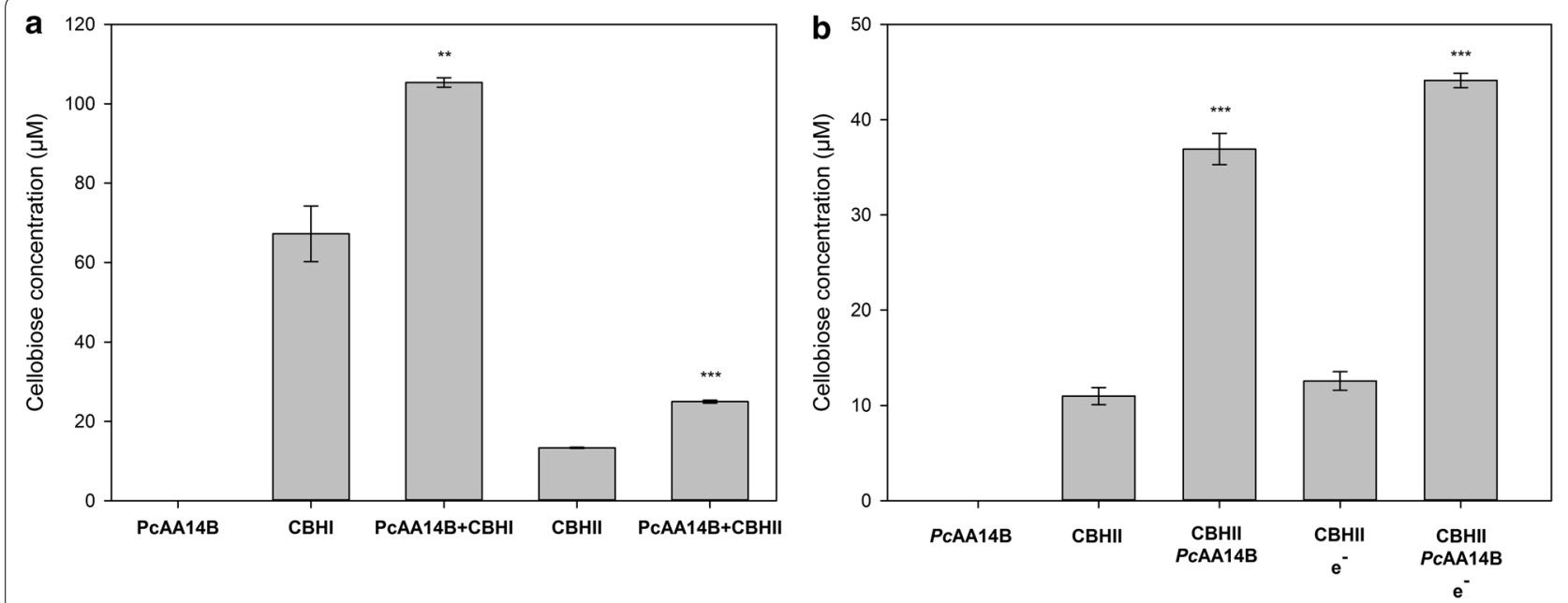

Fig. 4 Synergism between PCAA14B and cellobiohydrolases. a Synergistic effect of PCAA14B supplementation on CBH-mediated hydrolysis of substrate 1. b Synergistic effect of PCAA14B supplementation, with and without electron donor, on CBHII-mediated hydrolysis of microcrystalline cellulose (Avicel). Error bars represent the standard deviation from three independent experiments. Asterisks $\left({ }^{* *} p<0.01 ;{ }^{* * *} p<0.001\right)$ indicate significance based on Student's $t$ test

among filamentous fungi revealed that AA14 and GH30_7 genes can both be found at the genomic level in some well-known fungal saprotrophs like Trichoderma reesei, Podospora anserina and Fusarium oxysporum (Fig. 5), suggesting that such synergistic activities could occur in nature.

The synergistic effects observed between PcAA14B and TtXyn30A on birchwood cellulosic fibers were

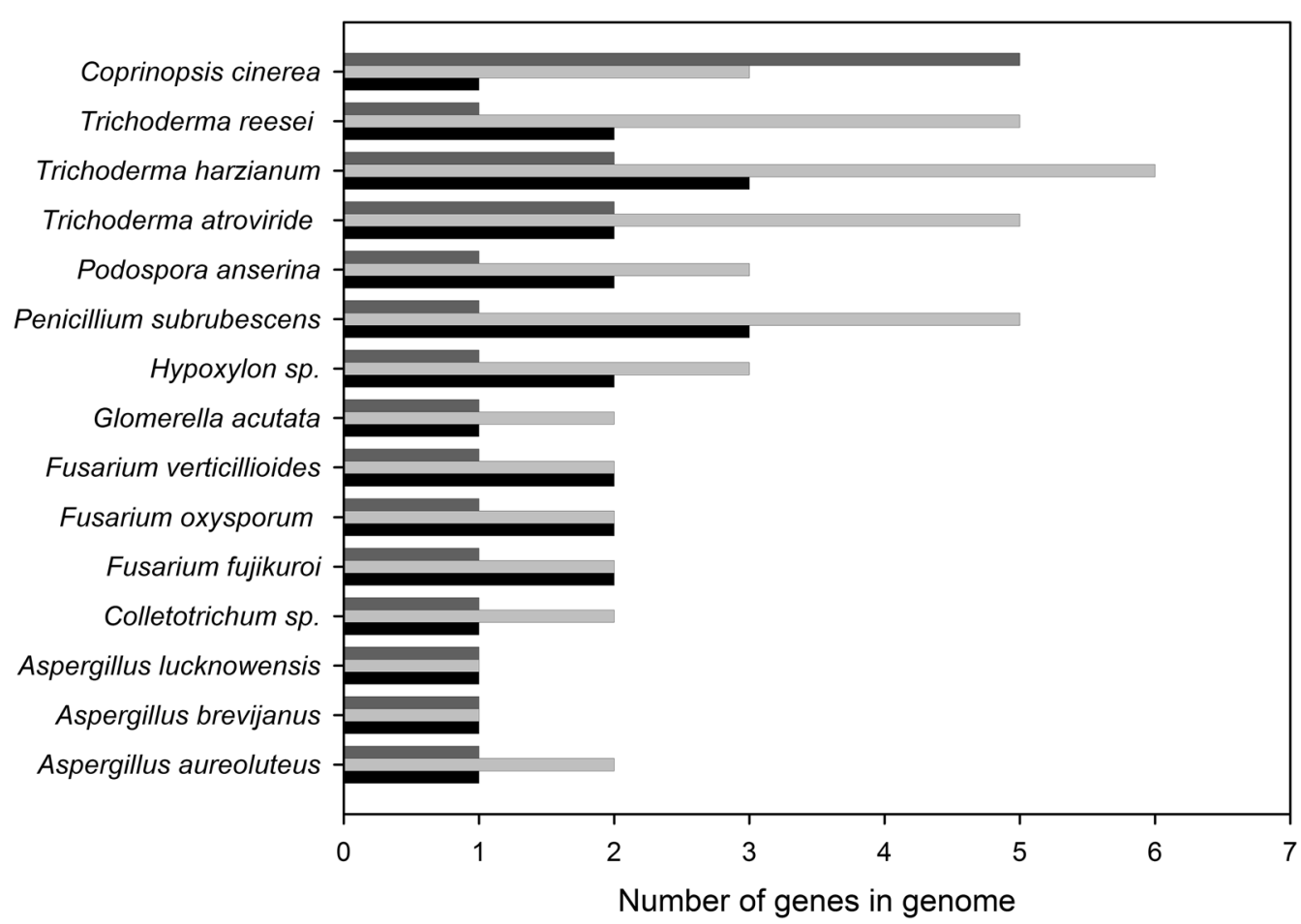

Fig. 5 Number of genes belonging to either GH30 (light grey bars), GH30_7 (black bars) or AA14 (dark grey bars) CAZy (sub)family, in fungal genomes 
significantly higher than those reported for $P c$ AA14B and a GH11 xylanase AnXyn11, as described by Couturier et al. [4] and repeated here for comparative purposes. The synergistic interaction between PcAA14B and TtXyn30A was found to be dose-dependent, while the addition of $P c$ AA14B led to ongoing xylobiose release by $T t \mathrm{Xyn} 30 \mathrm{~A}$ over a 24-h period, in contrast to the plateau reached by TtXyn30A only after $8 \mathrm{~h}$, during hydrolysis of birchwood cellulosic fibers. Altogether these results led to the hypothesis that the action of PcAA14B is constantly creating substrate sites available for $T t$ Xyn30A on the xylan polymer, as shown in Fig. 6.
The activity of TtXyn30A was tested in pretreated natural lignocellulosic substrates for the first time in this study. TtXyn30A also hydrolyzed the glucuronoxylan of most tested beechwood substrates with hemicellulose content $>10 \%(w / w)$, releasing xylobiose. A single exception to this was observed during hydrolysis of substrate 6, which contained high levels of residual lignin. Residual lignin was found to inhibit TtXyn30A. Although cellulases inhibition by the presence of lignin, due to enzyme absorption on the surface of the polymer [23], is much more prominent, xylanase activity was also reported to be negatively affected $[24,25]$, probably due to ionic

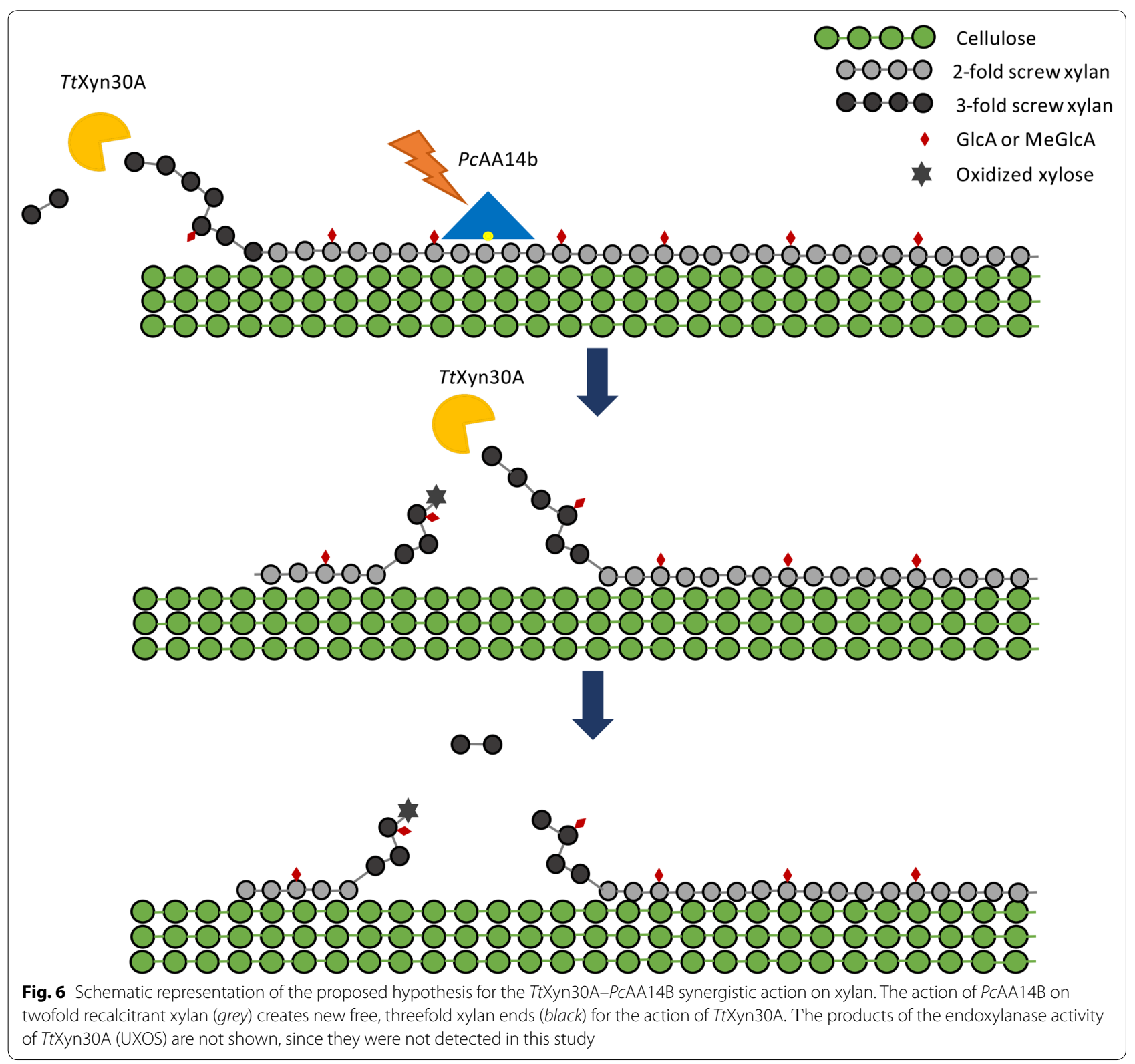


interactions between the charged moieties of the enzyme surface and lignin $-\mathrm{COOH}$ and $-\mathrm{CO}$ groups.

Regarding the activity of PcAA14B, Couturier et al. proposed a specific hypothesis for the substrate preference of this LPMO [4]; PcAA14B would be only active on the twofold screw xylan polymer that is attached to cellulose. Simmons et al. showed that the attachment of xylan polymers to underlying cellulose fibrils induces the twofold screw conformation on the xylan molecule, in contrast with the threefold screw conformation of soluble xylan [26]. Twofold screw xylan is a more rigid structure, resembling the cellulose fibrils, and therefore shows a certain degree of 'crystallinity' [6]. The results of our study strengthen the initial hypothesis, revealing a relationship between the hemicellulose content of the substrate, and the degree of synergism between the two enzymes; the lower the hemicellulose content, the higher the degree of synergism observed, at least for beechwood substrates, rich in glucuronoxylan. Moreover, the synergism among the two enzymes is less obvious in substrates with high hemicellulose content (e.g., 29\% w/w), where the recalcitrant part of xylan is perhaps not accessible to PcAA14B.

The high degree of synergism observed for cellulosic fibers and some of the tested beechwood substrates among PcAA14B and TtXyn30A, especially when compared to the results with $P c$ AA14B and AnXyn11 xylanase, can also be justified by previous findings on the conformation of xylan in plant cell walls. Grantham et al. previously showed that xylan decorations, such as acetyl, arabinosyl and GlcA or 4-O-MeGlcA groups, are essential for the proper attachment of xylan to the underlying cellulose fibrils, and therefore, for the formation of the twofold xylan structure [6]. The oxidative cleavage of recalcitrant xylan by $P c$ AA14B created free ends, which in the case of glucuronoxylan are evenly decorated with 4-O-MeGlcA moieties. The newly created free ends function as substrate sites for the xylobiohydrolase activity of $T t$ Xyn30A, which ultimately releases xylobiose. The synergistic effect is further supported by the complementary action of both enzymes: while PcAA14B oxidizes at the $\mathrm{C} 1$ position of the xylan backbone, $T t \mathrm{Xyn} 30 \mathrm{~A}$ releases xylose dimers from the non-reducing end. This synergistic relationship is similar to the synergism observed between CBHs and cellulose-active LPMOs during cellulose hydrolysis [13]. Products of the endoxylanase activity of TtXyn30A, such as 4-O-MeGlcA decorated $\mathrm{XOS}$, were detected in higher enzyme loadings, and in hemicellulose-rich substrates. In regard to the endoxylanase activity, TtXyn30A has been shown to attack the second glycosidic bond from the 4-O-MeGlcA position, while GH11 xylanases are well known to act several xylose monomers away from the substituent position, due to the architecture of the binding site cleft, which is not large enough to accommodate decorated xylans [27, 28]. This is also showed by Couturier et al., where the non-oxidized products found by the combined action of PcAA14B and $A n X y n 11$ were $\mathrm{X}_{3} \mathrm{MeGlcA}, \mathrm{X}_{4} \mathrm{MeGlcA}$ and $\mathrm{X}_{5} \mathrm{MeGlcA}$ [4].

The synergism observed between $T t \mathrm{Xyn} 30 \mathrm{~A}$ and PcAA14B was found to be more prominent when both enzymes were added simultaneously rather than sequentially. This is not usual for enzymes acting on the same polymer, due to competition for binding sites. However, there are some reports showing a similar trend [29]. Simultaneous addition of enzymes with complementary activities usually is more favorable when the enzymes act on different polymers (e.g., cellulases and xylanases) [30]. Malgas et al. proposed an 'alternating effect' for the synergism displayed among cellulases and xylanases, where each enzyme hydrolyzes the cellulose and xylan moieties, covering each other in the complex biomass structure [31]. This seems to be the case for the synergism among TtXyn30A and PcAA14B, as shown in Fig. 6. Time dependence experiments provide valuable insights regarding the mode of action of $P c A A 14 \mathrm{~B}$ and its synergism with $T t \mathrm{Xyn} 30 \mathrm{~A}$. When the substrate was pretreated with the LPMO and xylanase was added, after LPMO deactivation, for $24 \mathrm{~h}$, the xylobiose release was the same as in the case where both enzymes worked together for $24 \mathrm{~h}$. These data indicate that PcAA14B action modifies the substrate of $T t \mathrm{Xyn} 30 \mathrm{~A}$, making it more susceptible to hydrolysis, similarly to cellulose-acting LPMOs and cellobiohydrolases [13]. According to our hypothesis, although both enzymes act on the same polymer, its conformation is not the same: PcAA14B acts on the twofold helical screw xylan, creating free ends for TtXyn30A action, while TtXyn30A acts on the free non-reducing ends of the xylan polymer, due to its xylobiohydrolase activity. This synergistic relationship, aside from its obvious significance in the design of next-generation (hemi) cellulolytic cocktails, could also be employed for the production of oligosaccharides from residual biomass, which are shown to have significant prebiotic activity [32].

In the present work, the synergistic action of PcAA14B and cellobiohydrolases CBHI and CBHII was demonstrated for the first time. As previously shown, PcAA14B was found to enhance the glucose release from a (hemi) cellulolytic cocktail from $T$. reesei, but the synergism was not further studied with single enzymes [4]. Cellobiose release was found to be enhanced by the addition of PcAA14B on substrate hydrolysis by CBHs, and this finding in is accordance with the previous results, where the disruption of wood fibers was observed by the action of PcAA14B [4]. The authors hypothesized that the disruption of wood fibers revealed more substrate sites 
for cellulases, and the results of this work support this hypothesis. Moreover, the synergism between PcAA14B and CBHII was shown for Avicel degradation. This synergistic effect on Avicel can also be attributed to traces of recalcitrant xylan [33], the removal of which may facilitate cellulose hydrolysis by cellobiohydrolases, as proposed by Igarashi et al. [34].

\section{Conclusions}

In the present study, new synergistic relationships were established: the xylan-active LPMO, represented in this study by PcAA14B, and a novel GH30_7 glucuronoxylan-active xylanase with xylobiohydrolase activity, represented by $T$ tXyn30A, were found to act synergistically on cellulosic substrates. Also, the activity of TtXyn30A was documented against pretreated lignocellulosic substrates for the first time. The addition of $P c A A 14 B$ in the reaction mixture together with TtXyn30A increased greatly the xylobiose release from pretreated lignocellulosic substrates. The synergistic effects were most prominent in substrates of relatively low hemicellulose content, indicating a preference of $P c$ AA14B for recalcitrant xylan. We hypothesized that the action of PcAA14B on recalcitrant xylan creates new free ends in the polymer, which can be used as substrate for the action of TtXyn30A. PcAA14B was also found to act synergistically with cellobiohydrolases CBHI and CBHII. Overall, the results of the present study provide additional insights into the mode of action of the PcAA14B LPMO and will contribute to the design of next-generation enzymatic cocktails for the saccharification of woody lignocellulosic substrates with residual recalcitrant xylan.

\section{Methods}

\section{Enzymes and substrates}

Beechwood xylan, xylo-oligosaccharides (XOS), CBHI and CBHII cellobiohydrolases from Trichoderma longibrachiatum and a microbial source, respectively, and GH11 endo-1,4- $\beta$ - xylanase M4 from Aspergillus niger (AnXyn11), were purchased from Megazyme (Bray, Co. Wicklow, Ireland). Kraft birchwood cellulosic fibers (79\% (w/w) cellulose, 21\% (w/w) xylan, [4]) were kindly provided by Sandra Tapin (FCBA, Grenoble, France). Aqueous dispersions of Kraft birchwood cellulosic fibers were adjusted to $\mathrm{pH} 5.2$ with acetate buffer $(50 \mathrm{mM})$ in a final reaction volume of $0.5 \mathrm{~mL}$. Different beechwood lignocellulosic substrates were produced from commercially available beechwood (Lignocel ${ }^{\circledR}$ HBS 150-500), pretreated, as previously described [19]. TtXyn30A was produced and purified as described in [2]. PcAA14B was produced and purified, as previously described [4]. In brief, $P$. pastoris clones bearing the recombinant proteins in frame with (His) ${ }_{6}$-tag sequences were grown overnight at $30{ }^{\circ} \mathrm{C}$ in BMGY medium. The cells were then transferred to BMMY medium supplemented with methanol, as described in EasySelect ${ }^{\mathrm{TM}}$ Pichia Expression kit, and incubated 3-6 days for enzyme production. Then, the supernatants were collected by centrifugation, and they were filtered, concentrated and dialyzed. Both enzymes were purified with immobilized metal ion affinity chromatography (IMAC), to homogeneity.

\section{Bioinformatics analyses}

Bioinformatics analyses were performed on sequenced fungal genomes publically available (NCBI non-redundant sequence database and JGI). The co-occurrence of GH30_7 and AA14 modules in fungal genomes was searched using CAZy tools (www.cazy.org).

\section{Synergism assays}

Cellulosic fibers, lignocellulosic substrates, or Avicel were added to $50 \mathrm{mM}$ sodium acetate buffer, $\mathrm{pH} 5.2$ at a final concentration of $0.5 \%(\mathrm{w} / \mathrm{v})$. The enzymes were added at a final concentration of $2 \mu \mathrm{M}$ for PcAA14B and $0.1 \mu \mathrm{M}$ for TtXyn30A, AnXyn11, CBHI or CBHII, unless stated otherwise. Cysteine was used as an electron donor on the assays using birchwood cellulosic fibers and Avicel, at a final concentration of $1 \mathrm{mM}$. The reactions $(0.5 \mathrm{~mL})$ were incubated for $16 \mathrm{~h}$ in $45^{\circ} \mathrm{C}$, at $850 \mathrm{rpm}$, in a thermomixer (Eppendorf, Montesson, France). The reaction was terminated with 10 -min incubation in $100{ }^{\circ} \mathrm{C}$, and the reactions were centrifuged to remove the insoluble fraction.

The hydrolysis products were analyzed with high-performance anion-exchange chromatography (HPAEC), using a CarboPac PA-1 $(4 \times 250 \mathrm{~mm}$, Dionex $)$ column with a pulsed amperometric detector (PAD) equipped with a gold electrode. The solvents used were $\mathrm{NaOH}$ $100 \mathrm{mM}$ (solvent A) and $\mathrm{NaOAc}(1 \mathrm{M})$ in $100 \mathrm{mM} \mathrm{NaOH}$

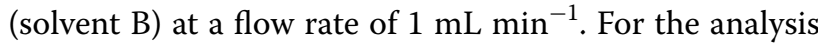
of oligosaccharides, the following gradient was applied: 0-10 min 0-10\% B, 10-35 min 10-30\% B, 35-40 min 30-100\% B 40-41 min 100-0\% B, 41-50 min isocratic step, $100 \%$ A. Xylobiose, XOS and cellobiose were quantified against suitable standard curves.

The degree of synergism was calculated according to the following equation:

$$
\mathrm{DS}=\frac{\mathrm{X}_{\mathrm{A}, \mathrm{B}}}{\mathrm{X}_{\mathrm{A}}+\mathrm{X}_{\mathrm{B}}}
$$

where $X_{A, B}$ is the $\mu \mathrm{M}$ of product from the action of both enzymes on the substrate, $X_{A}$ the $\mu \mathrm{M}$ of product from the action of the LPMO and $X_{B}$ the $\mu \mathrm{M}$ of product from the action of xylanases TtXyn30A or AnXyn11, or cellobiohydrolases $\mathrm{CBHI}$ and $\mathrm{CBHII}$.

The time dependence of the synergism between TtXyn30A and PcAA14B was tested, as mentioned above. 
First, a pretreatment of $10 \mathrm{mg} \mathrm{mL}^{-1}$ of substrate 1 was performed with either TtXyn30A $(0.1 \mu \mathrm{M})$ or PcAA14B $(2 \mu \mathrm{M})$ at $45{ }^{\circ} \mathrm{C}$ for $24 \mathrm{~h}$. Then, the first enzyme was deactivated by boiling the reaction mixture, the second enzyme was added (PcAA14B or TtXyn30A), and the reaction was incubated for a further $24 \mathrm{~h}$. The effect of TtXyn30A alone and together with PcAA14B added together from the start of the reaction and incubated for 24 or $48 \mathrm{~h}$ were used as controls. Xylobiose concentrations were determined with HPAEC-PAD, as mentioned above.

The synergism of PcAA14B with cellobiohydrolases $\mathrm{CBHI}$ and $\mathrm{CBHII}$ was studied, as mentioned above. In brief, $\mathrm{CBHI}$ and $\mathrm{CBHII}$ were added at a final concentration of $0.1 \mu \mathrm{M}$, while PcAA14B was added at a final concentration of $1 \mu \mathrm{M}$, to $15 \mathrm{mg} \mathrm{mL}^{-1}$ of substrate 1 . Cysteine $(1 \mathrm{mM})$ was added as electron donor, as indicated. The reaction was incubated at $45^{\circ} \mathrm{C}$ for $16 \mathrm{~h}$, and then the samples were boiled and filtered before HPAECPAD analysis. Cellobiose quantification was performed against a suitable calibration curve.

\section{Detection of UXOS in the reaction supernatants of $T$ XXyn30A}

For the detection of UXOS products of TtXyn30A, $50 \mathrm{mg} \mathrm{mL}^{-1}$ substrate 1 and 4 were treated with $0.25 \mathrm{mg} \mathrm{mL}^{-1} \mathrm{TtXyn} 30 \mathrm{~A}$ for $24 \mathrm{~h}$ in $50^{\circ} \mathrm{C}$ and $1100 \mathrm{rpm}$. Beechwood xylan $\left(5 \mathrm{mg} \mathrm{mL}{ }^{-1}\right)$ was hydrolyzed with $0.02 \mathrm{mg} \mathrm{mL}{ }^{-1} \operatorname{TtXyn} 30 \mathrm{~A}$ in the same conditions. The reactions were terminated by boiling the samples. The samples from hydrolysis of substrates 1 and 4 were diluted 2 times before HPAEC-PAD analysis, while the samples from beechwood xylan hydrolysis were diluted 10 times before analysis. HPAEC-PAD analysis was performed with a different elution program [2]. The solvents used were $\mathrm{NaOH} 100 \mathrm{mM}$ (solvent A) and $\mathrm{NaOAc}$ $(0.5 \mathrm{M})$ in $100 \mathrm{mM} \mathrm{NaOH}$ (solvent B) at a flow rate of

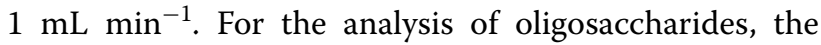
following gradient was applied: $0-5 \mathrm{~min} \mathrm{A,} \mathrm{5.1-30} \mathrm{min}$ 0-20\% B, 30.1-35 min 100\% B, 35.1-50 min 100\% A.

\section{Stability measurements}

The stability of PcAA14B and TtXyn30A was determined as follows: both enzymes were incubated in sodium acetate buffer, $50 \mathrm{mM}$, pH 5.2 for $48 \mathrm{~h}$ either in 4 or $45^{\circ} \mathrm{C}$. The final concentrations of enzymes were $2 \mu \mathrm{M}$ for PcAA14B and 0.1 $\mu \mathrm{M}$ for TtXyn30A. Samples were withdrawn in time points 0,24 and $48 \mathrm{~h}$, and the activity was determined. For TtXyn30A, the activity was measured with beechwood xylan as substrate, at a final concentration of $0.5 \%(\mathrm{w} / \mathrm{v})$, for $24 \mathrm{~h}$ in $45^{\circ} \mathrm{C}$ and $1200 \mathrm{rpm}$. The reducing sugars were quantified with the dinitrosalicylic acid method (DNS, [35]). In addition, the stability of TtXyn30A was measured also in the presence of $10 \mathrm{mg} \mathrm{mL}^{-1}$ substrate 1.

For PcAA14B, due to the lack of soluble products, the activity was determined by measuring the degree of synergism with TtXyn30A. In the PcAA14B-containing samples, substrate 1 was added at a final concentration of $10 \mathrm{mg} \mathrm{mL}^{-1}$ and fresh TtXyn30A at a final concentration of $0.1 \mu \mathrm{M}$. The mixture was incubated at $45^{\circ} \mathrm{C}$, and $1200 \mathrm{rpm}$ for $24 \mathrm{~h}$, and the xylobiose concentration was quantified with HPAEC-PAD, as described previously.

\section{Laccase treatment}

A treatment with laccase enzyme was performed in substrate 6 (Table 1). Laccase Novozym ${ }^{\circledR} 51003$ was used at $10 \mathrm{U} \mathrm{g}^{-1}$ substrate, which was added at a final concentration of $10 \%(\mathrm{w} / \mathrm{v})$. The mixture was incubated for $24 \mathrm{~h}$ in $40{ }^{\circ} \mathrm{C}, \mathrm{pH} 6,950 \mathrm{rpm}$, and then the supernatant was removed. The laccase-treated substrate was washed three times with ultrapure water, to remove low-MW phenolic compounds released by the action of the enzyme, and freeze-dried. Finally, it was hydrolyzed by TtXyn30A, as described previously. TtXyn30A was added at a final concentration of $1 \mu \mathrm{M}$.

\section{Statistical analysis}

All experiments were performed in triplicate, and the results are expressed as mean value \pm standard deviation. The statistical analysis was performed with SigmaPlot 12.5 software. Student's $t$ test was applied to experiment subsets, with the use of GraphPad Prism 5 software. $P$ values less than 0.05 were considered statistically significant.

\section{Supplementary information}

Supplementary information accompanies this paper at https://doi. org/10.1186/s13068-020-01777-x.

Additional file 1: Table S1. Xylo-oligosaccharide release from cellulosic fibers, after hydrolysis with PCAA14B, TtXyn30A, AnXyn11 and their combinations, as shown in Fig. 1. Error bars represent the standard deviation from three independent experiments. Figure S1. Analysis of XOS release by PCAA14B and TtXyn30A, in different pretreated lignocellulosic substrates, as shown in Table 1; (a) Substrate 5, (b) substrate 2, (c) substrate 4. Green line: substrate only, black line: PCAA14B, red line: TtXyn30A, blue line: PCAA14B and TtXyn30A. DP2: xylobiose. Figure S2. Analysis of XOS release by $T t X y n 30 A$, in beechwood xylan (10X diluted, blue line: reaction, light blue line: substrate blank) and substrate 4 (2X diluted, green line: reaction, yellow line: substrate blank); red line: 23-(4-O-Methyl-a-DGlucuronyl)-xylotriose, black line: XOS standards (DP 1-5).

\section{Acknowledgements}

The authors are grateful to K. G. Kalogiannis and A. A. Lappas from CPER (Thessaloniki, Greece) who kindly provided the pretreated biomass samples and to Novozymes A/S for the generous gift of Novozym ${ }^{\circledR} 51003$. The CAZy team, most especially Elodie Drula (AFMB, Marseille) are thanked for 
the functional annotation of fungal genomes. A.Z. thanks the French Embassy in Greece, together with the French Ministry of Higher Education, Research and Innovation, for the scholarship 'Séjours scientifiques de haut niveau' (SSHN).

\section{Authors' contributions}

AZ designed and performed experiments, analyzed data and wrote the original draft, with input from all authors; CP performed experiments and analyses; SG produced and purified enzymes and performed analyses; JGB and ET designed and supervised the study, and wrote the manuscript. All authors read and approved the final manuscript.

\section{Funding}

This study did not receive any particular funding.

\section{Ethics approval and consent to participate}

Not applicable.

\section{Consent for publication}

Not applicable.

\section{Competing interests}

The authors declare that they have no competing interests.

Received: 11 May 2020 Accepted: 29 July 2020

Published online: 10 August 2020

\section{References}

1. Vaaje-Kolstad G, Westereng B, Horn SJ, Liu Z, Zhai H, Sorlie M, Eijsink VG. An oxidative enzyme boosting the enzymatic conversion of recalcitrant polysaccharides. Science. 2010;330(6001):219-22. https://doi. org/10.1126/science.1192231.

2. Katsimpouras C, Dedes G, Thomaidis NS, Topakas E. A novel fungal GH30 xylanase with xylobiohydrolase auxiliary activity. Biotechnol Biofuels. 2019;12(1):120. https://doi.org/10.1186/s13068-019-1455-2.

3. Nakamichi Y, Fouquet T, Ito S, Watanabe M, Matsushika A, Inoue H. Structural and functional characterization of a bifunctional GH30-7 xylanase $B$ from the filamentous fungus Talaromyces cellulolyticus. J Biol Chem. 2019;294(11):4065-78. https://doi.org/10.1074/jbc.RA118.007207.

4. Couturier M, Ladevèze S, Sulzenbacher G, Ciano L, Fanuel M, Moreau C, Villares A, Cathala B, Chaspoul F, Frandsen KE, Labourel A, HerpoëlGimbert I, Grisel S, Haon M, Lenfant N, Rogniaux H, Ropartz D, Davies GJ, Rosso MN, Walton PH, Henrissat B, Berrin JG. Lytic xylan oxidases from wood-decay fungi unlock biomass degradation. Nat Chem Biol. 2018;14:306. https://doi.org/10.1038/nchembio.2558.

5. Busse-Wicher M, Grantham NJ, Lyczakowski JJ, Nikolovski N, Dupree P. Xylan decoration patterns and the plant secondary cell wall molecular architecture. Biochem Soc Trans. 2016;44:1. https://doi.org/10.1042/BST20 150183.

6. Grantham NJ, Wurman-Rodrich J, Terrett OM, Lyczakowski JJ, Stott K, luga D, Simmons TJ, Durand-Tardif M, Brown SP, Dupree R, Busse-Wicher M, Dupree P. An even pattern of xylan substitution is critical for interaction with cellulose in plant cell walls. Nat Plants. 2017;3:11. https://doi. org/10.1038/s41477-017-0030-8.

7. Terrett OM, Lyczakowski JJ, Yu L, luga D, Franks WT, Brown SP, Dupree R, Dupree P. Molecular architecture of softwood revealed by solid-state NMR. Nat Commun. 2019;10:4978. https://doi.org/10.1038/s41467-01912979-9.

8. Frommhagen M, Sforza S, Westphal AH, Visser J, Hinz SWA, Koetsier MJ, van Berkel WJH, Gruppen H, Kabel MA. Discovery of the combined oxidative cleavage of plant xylan and cellulose by a new fungal polysaccharide monooxygenase. Biotechnol Biofuels. 2015;8:101. https://doi. org/10.1186/s13068-015-0284-1.

9. Hüttner S, Várnai A, Petrović DM, Bach CX, Kim Anh DT, Thanh VN, Eijsink VGH, Larsbrink J, Olsson L. Specific xylan activity revealed for AA9 lytic polysaccharide monooxygenases of the thermophilic fungus Malbranchea cinnamomea by functional characterization. Appl Environ Microbiol. 2019;85:e01408-01419. https://doi.org/10.1128/aem.01408-19.
10. Basotra N, Dhiman SS, Agrawal D, Sani RK, Tsang A, Chadha BS. Characterization of a novel Lytic Polysaccharide Monooxygenase from Malbranchea cinnamomea exhibiting dual catalytic behavior. Carbohydr Res. 2019;478:46-53. https://doi.org/10.1016/j.carres.2019.04.006.

11. Corrêa TLR, Júnior AT, Wolf LD, Buckeridge MS, dos Santos LV, Murakami MT. An actinobacteria lytic polysaccharide monooxygenase acts on both cellulose and xylan to boost biomass saccharification. Biotechnol Biofuels. 2019;12:117. https://doi.org/10.1186/s13068-019-1449-0.

12. Nakamichi $Y$, Fouquet $T$, Ito $S$, Matsushika A, Inoue H. Mode of action of GH30-7 reducing-end xylose-releasing exo-xylanase A (Xyn30A) from the filamentous fungus Talaromyces cellulolyticus. Appl Environ Microbiol. 2019. https://doi.org/10.1128/AEM.00552-19.

13. Song B, Li B, Wang X, Shen W, Park S, Collings C, Feng A, Smith SJ, Walton JD, Ding SY. Real-time imaging reveals that lytic polysaccharide monooxygenase promotes cellulase activity by increasing cellulose accessibility. Biotechnol Biofuels. 2018;11:41-41. https://doi.org/10.1186/ s13068-018-1023-1.

14. Olsen JP, Borch K, Westh P. Endo/exo-synergism of cellulases increases with substrate conversion. Biotechnol Bioeng. 2017;114(3):696-700. https ://doi.org/10.1002/bit.26179.

15. Song HT, Gao Y, Yang YM, Xiao WJ, Liu SH, Xia WC, Liu ZL, Yi L, Jiang ZB. Synergistic effect of cellulase and xylanase during hydrolysis of natural lignocellulosic substrates. Bioresour Technol. 2016;219:710-5. https://doi. org/10.1016/j.biortech.2016.08.035.

16. Hu J, Arantes V, Pribowo A, Saddler JN. The synergistic action of accessory enzymes enhances the hydrolytic potential of a "cellulase mixture" but is highly substrate specific. Biotechnol Biofuels. 2013;6(1):112. https://doi. org/10.1186/1754-6834-6-112.

17. Harris PV, Welner D, McFarland KC, Re E, Navarro Poulsen JC, Brown K, Salbo R, Ding H, Vlasenko E, Merino S, Xu F, Cherry J, Larsen S, Leggio LL. Stimulation of lignocellulosic biomass hydrolysis by proteins of glycoside hydrolase family 61: structure and function of a large, enigmatic family. Biochemistry. 2010;49:15. https://doi.org/10.1021/bi100009p.

18. Karnaouri A, Muraleedharan MN, Dimarogona M, Topakas E, Rova U, Sandgren M, Christakopoulos P. Recombinant expression of thermostable processive MtEG5 endoglucanase and its synergism with MtLPMO from Myceliophthora thermophila during the hydrolysis of lignocellulosic substrates. Biotechnol Biofuels. 2017;10:126. https://doi.org/10.1186/s1306 8-017-0813-1.

19. Katsimpouras C, Kalogiannis KG, Kalogianni A, Lappas AA, Topakas E. Production of high concentrated cellulosic ethanol by acetone/water oxidized pretreated beech wood. Biotechnol Biofuels. 2017;10(1):54. https ://doi.org/10.1186/s13068-017-0737-9.

20. Biely P, Singh S, Puchart V. Towards enzymatic breakdown of complex plant xylan structures: state of the art. Biotechnol Adv. 2016;34:7. https:// doi.org/10.1016/j.biotechadv.2016.09.001.

21. Hu J, Arantes V, Saddler JN. The enhancement of enzymatic hydrolysis of lignocellulosic substrates by the addition of accessory enzymes such as xylanase: is it an additive or synergistic effect? Biotechnol Biofuels. 2011;4:36. https://doi.org/10.1186/1754-6834-4-36.

22. Couturier M, Haon M, Coutinho PM, Henrissat B, Lesage-Meessen L, Berrin JG. Podospora anserina hemicellulases potentiate the Trichoderma reesei secretome for saccharification of lignocellulosic biomass. Appl Environ Microbiol. 2011;77:1. https://doi.org/10.1128/AEM.01761-10.

23. Fong $M$, Berrin JG, Paës $G$. Investigation of the binding properties of a multi-modular $\mathrm{GH} 45$ cellulase using bioinspired model assemblies. Biotechnol Biofuels. 2016;9:1. https://doi.org/10.1186/s13068-016-0428-y.

24. Berlin A, Balakshin M, Gilkes N, Kadla J, Maximenko V, Kubo S, Saddler J. Inhibition of cellulase, xylanase and $\beta$-glucosidase activities by softwood lignin preparations. J Biotechnol. 2006;125:2. https://doi.org/10.1016/j. jbiotec.2006.02.021.

25. Kellock M, Rahikainen J, Marjamaa K, Kruus K. Lignin-derived inhibition of monocomponent cellulases and a xylanase in the hydrolysis of lignocellulosics. Bioresour Technol. 2017;232:183-91. https://doi.org/10.1016/j. biortech.2017.01.072.

26. Simmons TJ, Mortimer JC, Bernardinelli OD, Pöppler AC, Brown SP, deAzevedo ER, Dupree R, Dupree P. Folding of xylan onto cellulose fibrils in plant cell walls revealed by solid-state NMR. Nat Commun. 2016;7:13902. https://doi.org/10.1038/ncomms13902. 
27. Paës G, Berrin JG, Beaugrand J. GH11 xylanases: structure/function/properties relationships and applications. Biotechnol Adv. 2012;30(3):564-92. https://doi.org/10.1016/j.biotechadv.2011.10.003.

28. Berrin JG, Juge N. Factors affecting xylanase functionality in the degradation of arabinoxylans. Biotechnol Lett. 2008;30:7. https://doi.org/10.1007/ s10529-008-9669-6.

29. Väljamäe P, Sild V, Nutt A, Pettersson G, Johansson G. Acid hydrolysis of bacterial cellulose reveals different modes of synergistic action between cellobiohydrolase I and endoglucanase I. Eur J Biochem. 1999:266(2):32734. https://doi.org/10.1046/j.1432-1327.1999.00853.x.

30. Murashima K, Kosugi A, Doi RH. Synergistic effects of cellulosomal xylanase and cellulases from Clostridium cellulovorans on plant cell wall degradation. J Bacteriol. 2003;185(5):1518-24. https://doi.org/10.1128/ JB.185.5.1518-1524.2003.

31. Malgas S, Thoresen M, van Dyk JS, Pletschke BI. Time dependence of enzyme synergism during the degradation of model and natural lignocellulosic substrates. Enzyme and Microbial Technol. 2017;103:1-11. https ://doi.org/10.1016/j.enzmictec.2017.04.007.

32. Yu X, Yin J, Li L, Luan C, Zhang J, Zhao C, Li S. Prebiotic potential of xylooligosaccharides derived from corn cobs and their in vitro antioxidant activity when combined with lactobacillus. J Microbiol Biotechnol. 2015;25:1084-92. https://doi.org/10.4014/jmb.1501.01022.

33. Várnai A, Siika-Aho M, Viikari L. Carbohydrate-binding modules (CBMs) revisited: reduced amount of water counterbalances the need for CBMs. Biotechnol Biofuels. 2013;6:30. https://doi.org/10.1186/1754-6834-6-30.

34. Igarashi K, Uchihashi T, Koivula A, Wada M, Kimura S, Okamoto T, Penttilä M, Ando T, Samejima M. Traffic jams reduce hydrolytic efficiency of cellulase on cellulose surface. Science. 2011;333:1279-82. https://doi. org/10.1126/science.1208386.

35. Miller GL. Use of dinitrosalicylic acid reagent for determination of reducing sugar. Anal Chem. 1959;31(3):426-8. https://doi.org/10.1021/ac601 $47 \mathrm{a} 030$.

\section{Publisher's Note}

Springer Nature remains neutral with regard to jurisdictional claims in published maps and institutional affiliations.
Ready to submit your research? Choose BMC and benefit from:

- fast, convenient online submission

- thorough peer review by experienced researchers in your field

- rapid publication on acceptance

- support for research data, including large and complex data types

- gold Open Access which fosters wider collaboration and increased citations

- maximum visibility for your research: over $100 \mathrm{M}$ website views per year

At BMC, research is always in progress.

Learn more biomedcentral.com/submissions 